\title{
THE EFFECT OF LOCAL OXYTOCIN DELIVERY ON HEALING OF BONE DEFECTS IN RABBITS
}

\author{
Asmaa A. Harfoush ${ }^{l} B D S$, Gehan M Elba' $P h D$, Hanaa M Aly'2 $P h D$, Magda M Saleh ${ }^{3} P h D$
}

\begin{abstract}
INTRODUCTION: Bone defects above critical size do not heal completely by itself and thus represent major clinical challenge to reconstructive surgery. Numerous bone substitutes have already been used to promote bone regeneration. Incorporation of 31 biologically active molecules to bone grafts have shown to further improve its bone forming capacity. As a potential candidate for bone regenerating biomolecules which can be used in promoting proliferation and osteogenic differentiation of mesenchymal stem cells, the hypothalamic hormone oxytocin is suggested to be promising.

AIM OF THE STUDY: was to evaluate the efficacy of the hypothalamic nonapeptide oxytocin (OT) by direct delivery into local defects using $\beta$-tricalcium phosphate ( $\beta$-TCP) as a carrier.

MATERIALS AND METHODS: Twenty one male New Zealand white rabbits weighing $3 \mathrm{Kg}( \pm 250 \mathrm{~g})$ were used in this study. Right and left critical size bone defects (CSD) were performed in the edentulous area of rabbit mandibles (diastema). Right side bone defects (Experimental) were filled with OT loaded $\beta$-TCP, while the left side bone defects (Control), were filled with saline loaded $\beta$-TCP. The effect of OT hormone on bone formation was assessed histologically as well as histomorphometrically after 1, 3 and 6 weeks.

RESULTS: Greater amount of new bone formation was noticed in the CSDs filled with the OT loaded groups. The amount of new bone formed was significantly higher during the 3 observational intervals in the OT loaded $\beta$-TCP than when compared with the saline loaded $\beta$ TCP.
\end{abstract}

CONCLUSION: OT induces new bone formation via an osteoinductive action and can be added to bone grafts following surgical and periodontal surgeries to enhance bone regenerative capacity.

KEYWORDS: bone graft; bone regeneration; histomorphometry; oxytocin

1 Demonstrator of Oral Biology - Faculty of Dentistry - Alexandria University.
2 Professor of Oral Biology - Faculty of Dentistry - Alexandria University.
3 Professor and Head of Oral and Maxillofacial Surgery Department-Faculty of Dentistry-Alexandria University.

\section{INTRODUCTION}

In the maxillofacial region, bone defects occur commonly as a result of periodontal diseases, infectious disorders, trauma, removal of cysts or tumors, and after orthognathic or implant surgery. Various techniques have been developed in order to reconstruct these bone defects with the use of autogenous bone grafts, bone derivatives or bone substitutes (1).There are more than 1 million bone graft operations are performed in the United States alone every year (2).

Autologous bone is the gold standard for the reconstruction of bone defects, however, there have been obstacles to its use such as the amount of bone to harvest is limited, and a second operation site increases patient morbidity (3). Bone allografts are alternatives, but they are expensive, and carry the risk of disease transmission. (4).For these reasons, the need for synthetic bone substitutes has increased dramatically (5). However, most synthetic bone grafts have limited capacity to reconstitute bone in large defects because they lack the osteoinductivity and osteogeneicity of autologous bone grafts. Attempts to remedy this by adding osteogenic growth factors absorbed onto a biomaterial such as synthetic bone grafts (6), injected locally or delivered systemically in order to enhance their bone forming capacity (7). Such incorporation of biological substances enhances new bone formation by promoting the proliferation and differentiation of osteoblast lineages $(8,9)$.

The microporous $\beta$-tricalcium phosphate (TCP) is one of the synthetic bone grafts, which has been successfully widely used as a delivery system of various biologically active molecules including bone morphogenetic proteins (BMP) and platelet-derived growth factor (PDGF), as a carrier to directly deliver OT to the sites of local defects (811)

Oxytocin (OT) is a hormone that has recently received much interest owing to its ability to promote bone formation and

thus, can be used as one of the bone regenerating biomolecules that can promote proliferation and the osteogenic differentiation of mesenchymal stem cells to mature mineralizing osteoblasts (10-12).

The neuropeptide oxytocin (OT) is mainly synthesized in the magnocellular neurons of the supraoptic and paraventricular nuclei of the hypothalamus and then transported to the posterior pituitary, where it is released into the periphery via the bloodstream. It has long been known to be related to parturition and milk ejection. However, OT has also been described in various additional functions, the most important of which is its role in bone formation $(12,13)$.

OT affects bone formation by modulating both osteogenesis and osteoclastogenesis in favor for bone formation. OT treatment favours osteogenesis, it stimulates osteoblast differentiation via an up-regulation of BMP-2 and osterix gene expression. Studies have suggested that the bone anabolic effect of OT is achieved by a peripheral mode of action $(10,14)$. On the other hand, it inhibits bone resorption by mature osteoclast by triggering cytosolic calcium ion release and nitric oxide synthesis (15).

It was verified that deletion of OT or OT receptors (OTR) in young rodents caused reduced bone formation and suggested that OT is indispensable for basal skeletal 
homeostasis in both sexes (15). In adult male albino rats intramuscular OT injection was found to have growth promoting effects on bone. Subcutaneous OT injection reversed bone loss in eight-week-old ovariectomized (OVX) mice and reduced marrow adiposity (11).

Considering the results of previous studies, it is clearly suggested that OT might be an effective molecule for enhancing bone healing of osseous defects. However, all of the studies reporting the bone anabolic activity of OT have only evaluated the bone healing effect in rodents via systemic OT administration by peritoneal or subcutaneous injection $(10,15)$.

Not enough studies have yet evaluated the effect of direct OT delivery on bone healing capacity of bone graft materials in bone regenerative procedures.

Aim of the study was to evaluate the efficacy of the hypothalamic nonapeptide oxytocin (OT) by direct delivery into local defects using $\beta$-tricalcium phosphate $(\beta$-TCP) as a carrier.

\section{Materials and Methods}

Animals \& experimental design:

The Ethical Committee of the Faculty of Dentistry Alexandria University approved the protocol of this research. Twenty one skeletally mature male New Zealand white rabbits, each weighing $3 \mathrm{~kg}( \pm 250 \mathrm{~g})$, were used in this study. The animals were obtained from the Institute of Medical Research Alexandria University. They were housed in specially designed wire mesh bottom cages, three rabbits per cage. The animals were supplied a regular diet during the whole experimental period.

Rabbits were randomly divided into 3 groups according to the observation period of 1,3 , and 6 weeks after surgery as follows:

Group I: (7 rabbits) sacrificed after 1 week.

Group II :( 7 rabbits) sacrificed after 3 weeks.

Group III :( 7 rabbits) sacrificed after 6 weeks.

Materials:

IngeniOs $\beta$-TCP Bioactive Synthetic Bone Particles (Zimmer Dental Inc., Carlsbad, CA) and OT acetate salt hydrate (Sigma-Aldrich, St. Louis, MO, USA) were purchased. OT acetate salt hydrate was reconstituted in physiological saline at a concentration of $2.5 \mathrm{mg} \mathrm{OT} / \mathrm{ml}$ saline (16).

\section{Surgical procedure}

General anesthesia was induced by an intramuscular injection of a combination of $25 \mathrm{mg} / \mathrm{kg}$ body weight ketamine hydrochloride (Ketamine ${ }^{\circledR} 50 \mathrm{mg} / \mathrm{ml}$ ) and $5 \mathrm{mg} / \mathrm{kg}$ body weight Xylazine hydrochloride (Xyla-Ject ${ }^{\circledR}$ ADWIA Co, 10th of Ramadan City, Egypt). The mandible was shaved on both sides, and the skin was rinsed and scrubbed with 2\% Povidone-iodine (Betadine, Nile Co, Egypt). The oral commissures were widened by cutting through the corner of the mouth and past it by one centimeter for better surgical access. The rabbits have a permanent dentition with a dental formula of; I $2 / 1 \mathrm{C} 0 / 0 \mathrm{P} 3 / 2 \mathrm{M} 3 \beta=28$, the edentulous alveolar ridges of the body of the mandible (diastema) between the incisor and the first posterior tooth on both sides of each animal were selected for the surgical procedure. Full mucoperiosteal flaps were raised intraorally on both sides of the mandible in each animal. Critical size osseous defects measuring $5 \mathrm{~mm}$ length $\times 3 \mathrm{~mm}$ width $\times 3 \mathrm{~mm}$ depth were prepared in the edentulous left and right regions of the alveolar ridge of the mandible, using a sterile surgical bur under water cooling system (17).
The osseous defects performed in each animal were washed out with sterile saline before loading. Right sided osseous defects (experimental side) were filled with Oxytocin loaded $\beta$-TCP, while left sided bone defects (control side) were filled with saline loaded $\beta$-TCP $(16,17)$.

Post-operatively, each animal received the same course of antibiotic of $25 \mathrm{mg} / \mathrm{kg}$ body weight Oxytetracycline (PAN Terramycin ${ }^{\circledR}$, Pfizer, Egypt) for five days after surgery every eight hours. Analgesics were given to animals in the form of diclofenac sodium IM (Declophen ${ }^{\circledR}$, Pharco Pharmaceuticals, Egypt) every eight hours for the first two days. The animals were observed daily for the first week to assess the occurrence of any signs of inflammation or infection.

Euthanasia was done after 1, 3 and 6 weeks respectively by an overdose of the anesthetic solution Thiopentone Sodium injected rapidly. The mandibles were dissected out, sectioned into two halves and fixed in $10 \%$ neutral buffered formalin. After fixation, the mandibles were decalcified in $5 \%$ trichloroacetic acid, washed, dehydrated in ascending order of ethanol and embedded in paraffin wax. Serial bucco-lingual (sagittal) sections of $5 \mu \mathrm{m}$ thickness were cut and stained with Hematoxylin \& Eosin.

Histomorphometric analysis using Image J software was done to obtain the percentage of surface area of the formed bone compared to the total surface area of the created bone defect in the three studied observation periods of bone healing (18).

Five sections of tissue from different standardized depths were used for quantification from each sample. A rectangle with standardized dimensions was drawn on the desired area to be measured using the image $\mathrm{J}$ program. The surface area of this selected region was measured by choosing Region of Interest (ROI) manager, and the measurement was recorded. The surface area occupied by the marrow spaces was selected using the wand tracing tool and the measurement was recorded.

The two recorded measurements were subtracted to obtain the surface area occupied only by bone, and its percentage to the total area selected was calculated. The measurements from three photographs were recorded and their mean was calculated for each of the five sections obtained, from each specimen

Statistical analysis of the obtained data was done using paired t-test to compare the percentage of new bone formed in the OT and saline $\beta$-TCP loaded groups for each observational period.

\section{RESULTS}

1-Histological results

Results of group I, first observational period (one week post operatively):

A- Saline loaded $\boldsymbol{\beta}$-TCP (control group): The defects were filled with dense granulation tissue. $\beta$-TCP particles occupied the central part of the defect and were surrounded by the fibrous tissue. Newly formed woven bone was seen mainly extending from the lateral walls of the defect a short distance towards its center. A line of fusion could be seen between the native bone and the newly formed bone. (Figure 1) 


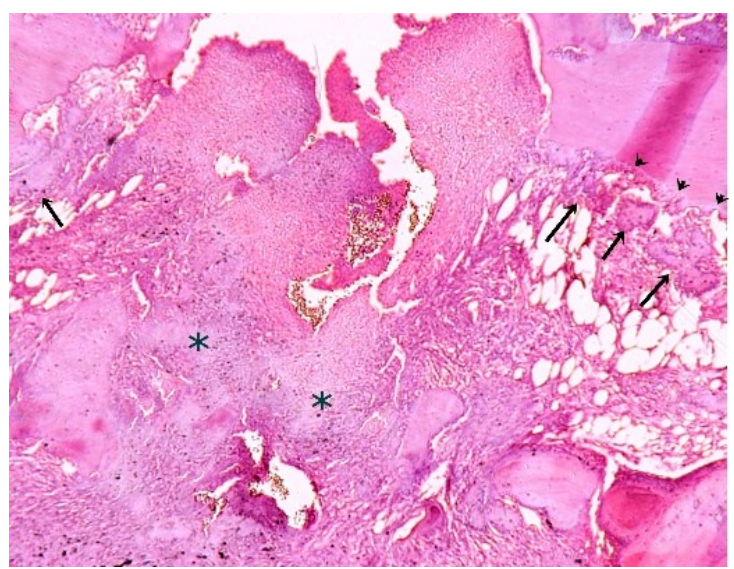

Fig.1: Light photomicrograph showing the bone defect (saline loaded $\beta$-TCP group, first week) filled with $\beta$-TCP particles (asterisks) surrounded by dense granulation tissue. New bone formation appears confined to the lateral wall of the defect (arrows). Note the line of fusion between old bone and new bone (arrow heads). H\&E stain original magnification $\mathrm{x} 40$.

B- Oxytocin loaded $\beta$-TCP (experimental group): Observations were different here in comparison to the control group. The granulation tissue formation was less and the newly formed bone also differed in terms of its quantity and organization. More new bone formation was observed in the areas close to the defect margins. Moreover, bony islands were detected in the central defect areas. The newly formed bone consisted of anastomosing bone trabeculae of moderate thickness. Graft particles were also seen in the central portion of the defect surrounded by fibrous tissue. Also, a line of fusion could be seen between native bone and the newly formed bone. (Figure 2)

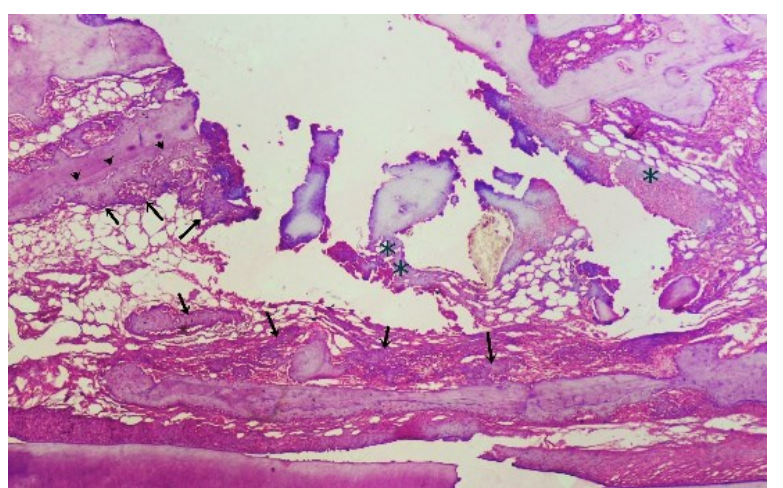

Fig.2: Light photomicrograph (oxytocin loaded $\beta$-TCP group, first week) showing more new bone formation (arrows) than that found in the control group. Formation of new bone extended from the lateral walls as well as at the center of the defect. $\beta$-TCP particles are also present (asterisks). Note the line of fusion between old bone and new bone (arrow heads). H\&E stain original magnification $\mathrm{x} 40$.

2. Results of group II, second observational period (three weeks post operatively):

Saline loaded $\boldsymbol{\beta}$-TCP group: The overall view of the defect showed that it was still filled with fibrous tissue. Newly formed bone masses were observed confined to the lateral walls of the defect but more than that formed after the first week. The $\beta$-TCP particles were seen surrounded by fibrous tissue. (Figure 3)

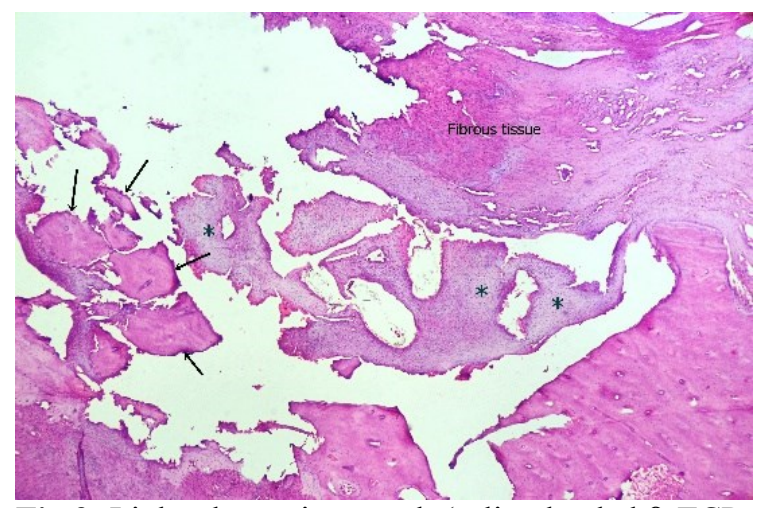

Fig.3: Light photomicrograph (saline loaded $\beta$-TCP group, third week) showing the majority of the defect filled with fibrous tissue surrounding $\beta$-TCP particles (asterisks) with few scattered new bone spicules (arrows) occupying the lateral wall of the defect. $\mathrm{H} \& \mathrm{E}$ stain original magnification $\mathrm{x} 40$.

Oxytocin loaded $\boldsymbol{\beta}$-TCP group: The entire defect was filled with new bone in the form of intercommunicating bone trabeculae enclosing bone marrow spaces of rich vascularity. The newly formed bone trabeculae showed different maturity degrees ranging from thin to more thick trabeculations of bone surrounded by minute amount of fibrous tissue. (Figure 4)

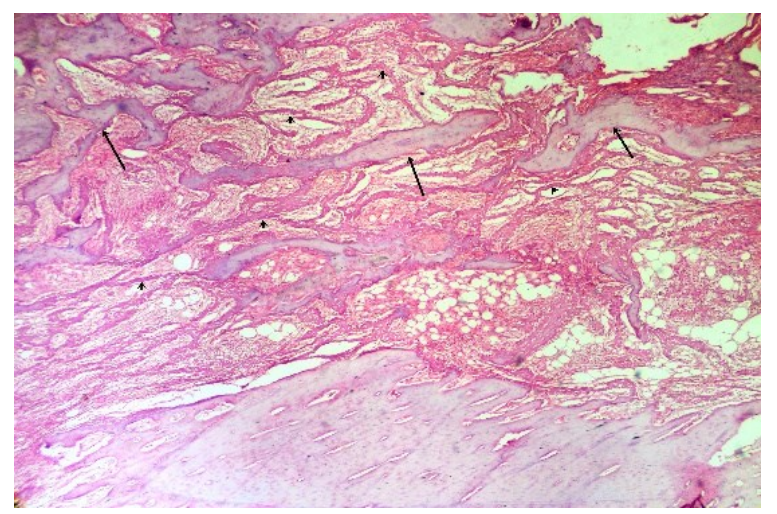

Fig.4: Light photomicrograph (Oxytocin loaded $\beta$-TCP group, third week) showing evident active bone formation. The defect is entirely filled with new bone of varying maturity ranging from thick bone trabeculae (arrows) to thin fibrous tissue mapping of collagen (arrow heads). H\&E stain original magnification $\mathrm{x} 40$

Results of group III, third observational period (six weeks post operatively):

A- Saline loaded $\boldsymbol{\beta}$-TCP group: The margins of the defect was sealed by compact bone while the central part was filled with trabecular bone of varying thickness and density surrounded by fibrous connective tissue. (Figure 5)

B- Oxytocin loaded $\boldsymbol{\beta}$-TCP group: The defects were almost filled with dense compact bone which appeared to be of higher density than in the control group while there was small areas of trabecular bone mass near the central part of the defect. The compact bone consisted of numerous Haversian systems enclosing rich blood supply within their Haversian canals. (Figure 6) 


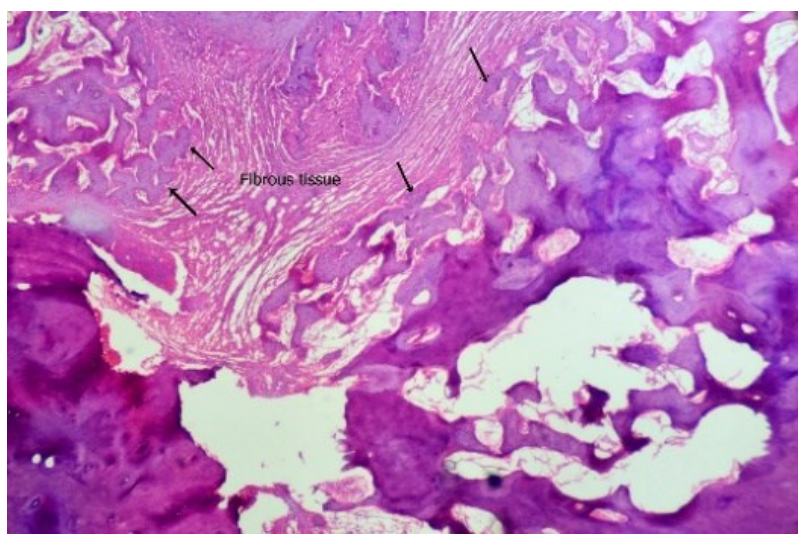

Fig.5: Light photomicrograph (saline loaded $\beta$-TCP group, 6 weeks) a considerable part of the defect is still filled with fibrous tissue at the center. Dense new bone formation is shown extending from the lateral wall while the center of the defect contains trabecular bone of varying thickness (arrows). H\&E stain original magnification $\mathrm{x} 40$.

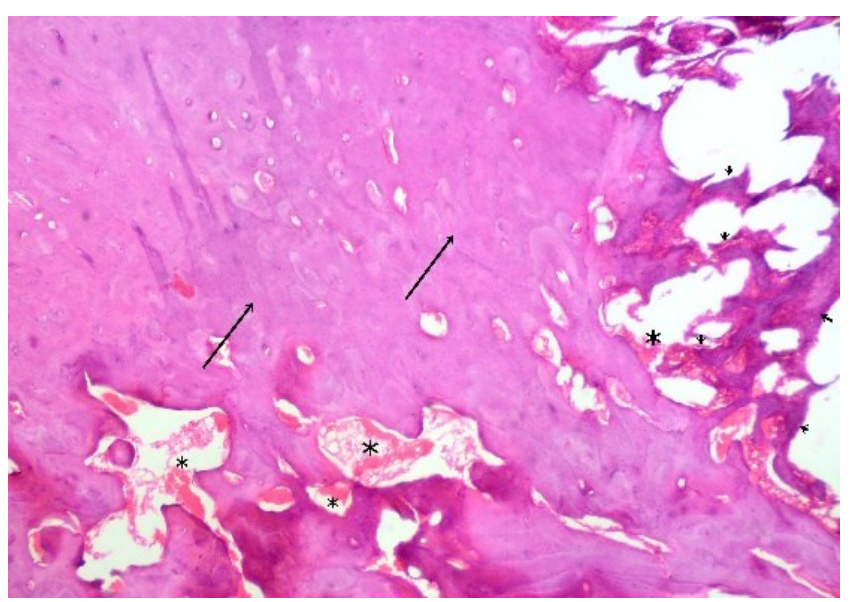

Fig.6: Light photomicrograph (Oxytocin loaded $\beta$-TCP group, 6 weeks) showing mature compact bone (arrows) at the marginal part of the defect with continuation of extension of the underlying trabecular bone (arrow heads) towards the central part of the defect. Note the presence of rich blood supply (asterisks) adjacent to the formed bone mass. H\&E stain original magnification $\mathrm{x} 40$

\section{Histomorphometric analysis}

The mean values of the percentages of bone surface area formed during the healing of the bone defect in the saline loaded $\beta$-TCP and OT loaded $\beta$-TCP groups after 1,3 , and 6 weeks were calculated and demonstrated in table (1).

Table 1: Percentage of the newly formed bone occupying the defect area.

\begin{tabular}{|l|l|l|l|}
\hline & $\begin{array}{l}\text { Saline-loaded } \\
\text { group }\end{array}$ & $\begin{array}{l}\text { OT-loaded } \\
\text { group }\end{array}$ & P value \\
\hline $\begin{array}{l}1 \text { week } \\
\text { Mean } \pm \text { SD }\end{array}$ & $10.49 \pm 3.08$ & $18.27 \pm 1.84$ & $0.002^{*}$ \\
\hline $\begin{array}{l}3 \text { weeks } \\
\text { Mean } \pm \text { SD }\end{array}$ & $29.76 \pm 4.80$ & $41.77 \pm 7.65$ & $0.01^{*}$ \\
\hline $\begin{array}{l}6 \text { weeks } \\
\text { Mean } \pm \text { SD }\end{array}$ & $65.39 \pm 7.86$ & $89.59 \pm 3.92$ & $0.001^{*}$ \\
\hline
\end{tabular}

*: Statistical significance $(\mathrm{p} \leq 0.05)$

The percentage of new bone formed (NB \%) is defined as the percentage of bone formed in the Region of Interest (ROI). At 1 week, the mean NB\% for the control group was
$10.49 \%$ and, $18.27 \%$ for the study group $(\mathrm{p}=0.002)$. While for the second observational period after 3 weeks, the NB\% for the control was $29.76 \%$ and, $41.77 \%$ for the study group $(p=0.01)$. At 6 weeks, NB\% for the control and the study group were $65.39 \%$ and $89.59 \%$ respectively $(p=0.001)$. Therefore, the OT loaded groups exhibited statistically significantly greater NB\% compared with the control groups in all of the three observational periods.

\section{DISCUSSION}

Oxytocin hormone is produced from the posterior pituitary gland and has recently shown its ability to act on bone cels, modifying their action to cause an increase in bone formation as well as an inhibition of bone resorption (15).

This study was performed in order to evaluate the effect of OT hormone on bone formation when it was locally delivered to mandibular bone defects in rabbit models using $\beta$-TCP bone graft as a carrier.

IngeniOs $\beta$-TCP bone graft was the material of choice to act as a delivery vehicle for the OT hormone in our study because of its highly porous nature of up to $70 \%$ porosity and of pore size $250-1000 \mu \mathrm{m}$ (19).

Our findings regarding the OT effect on new bone formation in critical size osseous defects show that the OT notably promoted bone formation especially during the early healing phase.

The new bone formed in the control groups (saline loaded $\beta$-TCP) was always lower than that found in the OT loaded $\beta$-TCP groups. The new bone formed in the experimental groups was higher, bony trabeculae was also thicker and the number of osteocyte content was notably larger than those found in the control group. These results are in agreement with the findings of Park et al (16) who also examined the effect of local OT delivery but in critical size calvarial bone defects of rats.

In another study performed by Colli et al (11) it was proven that systemic administration of OT promoted bone formation and inhibited bone resorption in the healing of extraction socket.

According to Elabd et al (10) OT promotes bone formation. It was shown that subcutaneous OT injection reverses bone loss in ovariectomized mice and enhances bone microarchitecture and biomechanical strength.

Colaianni et al (12) confirmed the OT action on bone when OT and OT-receptors null mice develop osteoporosis that worsens with age in both sexes. Histomorphometry revealed a pronounced decrease in trabecular volume accompanied by a significant reduction in bone formation rate. This bone deleterious effect could be rescued by intraperitoneal injection of OT.

Providing further confirmation to the positive action of OT on bone, Tamma et al (15) conducted a study where OT was injected intraperitoneal in mice and the results were an increase in bone mineral density as well as osteoblast differentiation.

Moreover, in a recent study performed on human beings Breuil et al (20) in which postmenopausal osteoporotic women with at least one bone fracture were compared with healthy controls, showed that low OT serum levels was significantly associated with severe osteoporosis. These results reinforce the concept that OT plays a role in the pathophysiology of postmenopausal osteoporosis.

The mode of action of the enhanced bone healing is apparently due to an induction of osteoblast differentiation 
from mesenchymal stem cells via OT release from the implanted $\beta$-TCP. It was noticed that the OT loaded groups exhibited new bone formation with dense osteoblast lining which appear voluminous and active, one can subsequently conclude that OT increases both osteoblast proliferation and differentiation, these results are in agreement with Colaianni et al and Tamma et al $(13,15)$ who examined the effect of OT on bone and stated that OT triggers osteoblast differentiation via an up regulation of BMP-2 as well as osteopontin (OPN) and osteocalcin (OCN) mRNAs expression in osteoblasts. Moreover, Colli et al (11) have reported that OT promotes a significant increase in alkaline phosphatase ALP and OCN which are excellent biochemical markers of bone formation activity.

On the other hand, OT inhibits bone resorption. Colaianni et al (12) supports this and explains that besides the bone forming capacity of OT, it may promote osteoclastic differentiation, yet, it prevents the resorptive action of mature osteoclasts, thus it tends to inhibit bone resorption. Maclntyre et al (21) explains the mechanism by which OT inhibits bone resorption and states that OT tends to cause a decrease of the RANKL/OPG ratio. Also, it causes the release of intracellular calcium leading to an increase of intracellular calcium level inside osteoclast cells which in turn increases the synthesis of nitric oxide as a mechanism to inhibit bone resorption.

Another interesting finding was the high vascularity to the defect area in the OT loaded groups compared to the control groups. This could be explained by Catteneo et al (22) in his study about the effect of OT on human endothelial cells, who proved that OT is a strong chemoattractant agent that causes proliferation and migration of endothelial cells and therefore promotes angiogenesis

The positive bone balance and the decreased bone resorption observed with the OT loaded $\beta$-TCP groups during the healing of the defects suggest that a simple and direct form of OT delivery promotes osteoinductive bone formation. Thus, local OT delivery would be promising as a method of achieving rapid and predictable bone regeneration of osseous defects in periodontal disease and implant related regenerative procedures.

\section{CONCLUSION}

Local OT administration may be effective for achieving favourable new bone formation in large osseous defects. Such approach is expected to provide improved clinical outcomes in the effort to restore large osseous defects in which osteoconductive bone substitutes have only limited bone healing capacity.

\section{CONFLICT OF INTEREST}

The authors declare that they have no conflict of interest.

\section{REFERENCES}

1. Podaropoulos L, Veis AA, Papadimitriou S, Alexandridis C, Kalyvas D. Bone regeneration using btricalcium phosphate in a calcium sulfate matrix. Journal of Oral Implantology. 2009;35(1):28-36.

2. Hutmacher DW. Scaffolds in tissue engineering bone and cartilage. Biomaterials. 2000;21(24):2529-43.

3. Jang ES, Park JW, Kweon H, Lee KG, Kang SW, Baek $\mathrm{D}-\mathrm{H}$, et al. Restoration of peri-implant defects in immediate implant installations by Choukroun plateletrich fibrin and silk fibroin powder combination graft.
Oral Surgery, Oral Medicine, Oral Pathology, Oral Radiology, and Endodontology. 2010;109(6):831-6.

4. Giannoudis PV, Dinopoulos H, Tsiridis E. Bone substitutes: an update. Injury. 2005;36(3):S20-S7.

5. Varghese S, Phadke A. Synthetic bone grafts. Google Patents; 2014.

6. Clarke S, Brooks R, Lee P, Rushton N. The effect of osteogenic growth factors on bone growth into a ceramic filled defect around an implant. Journal of Orthopaedic Research. 2004;22(5):1016-24.

7. Clarke S, Hoskins N, Jordan G, Henderson S, Marsh D. In vitro testing of Advanced JAX ${ }^{\mathrm{TM}}$ Bone Void Filler System: species differences in the response of bone marrow stromal cells to $\beta$ tri-calcium phosphate and carboxymethylcellulose gel. Journal of Materials Science: Materials in Medicine. 2007;18(12):2283-90.

8. Rodriguez R, Kondo H, Nyan M, Hao J, Miyahara T, Ohya K, et al. Implantation of green tea catechin $\alpha$ tricalcium phosphate combination enhances bone repair in rat skull defects. Journal of Biomedical Materials Research Part B: Applied Biomaterials. 2011;98(2):26371.

9. Xu L, Lv K, Zhang W, Zhang X, Jiang X, Zhang F. The healing of critical-size calvarial bone defects in rat with rhPDGF-BB, BMSCs, and $\beta$-TCP scaffolds. Journal of Materials Science: Materials in Medicine. 2012;23(4):1073-84.

10. Elabd C, Basillais A, Beaupied H, Breuil V, Wagner N, Scheideler M, et al. Oxytocin controls differentiation of human mesenchymal stem cells and reverses osteoporosis. Stem Cells. 2008;26(9):2399-407.

11. Colli VC, Okamoto R, Spritzer PM, Dornelles RCM. Oxytocin promotes bone formation during the alveolar healing process in old acyclic female rats. Archives of Oral Biology. 2012;57(9):1290-7.

12. Colaianni G, Tamma R, Di Benedetto A, Yuen T, Sun L, Zaidi M, et al. The Oxytocin-Bone Axis. Journal of Neuroendocrinology. 2014;26(2):53-7.

13. Colaianni G, Sun L, Di Benedetto A, Tamma R, Zhu L$\mathrm{L}$, Cao J, et al. Bone marrow oxytocin mediates the anabolic action of estrogen on the skeleton. Journal of Biological Chemistry. 2012;287(34):29159-67.

14. Elabd S, Sabry I, Hassan W, Nour H, Zaky K. Possible neuroendocrine role for oxytocin in bone remodeling. Endocrine Regulations. 2007;41(4):131-41

15. Tamma R, Colaianni G, Zhu Ll, DiBenedetto A, Greco $\mathrm{G}$, Montemurro $\mathrm{G}$, et al. Oxytocin is an anabolic bone hormone. Proceedings of the National Academy of Sciences. 2009;106(17):7149-54.

16. Park JW, Kim JM, Lee HJ, Jeong SH, Suh JY, Hanawa $\mathrm{T}$. Bone healing with oxytocin-loaded microporous $\beta$ TCP bone substitute in ectopic bone formation model and critical-sized osseous defect of rat. Journal of Clinical Periodontology. 2014;41(2):181-90.

17. Eleftheriadis E, Leventis MD, Tosios KI, Faratzis G, Titsinidis S, Eleftheriadi I, et al. Osteogenic activity of. BETA.-tricalcium phosphate in a hydroxyl sulphate matrix and demineralized bone matrix: a histological study in rabbit mandible. Journal of Oral Science. 2010;52(3):377-84.

18. Yugoshi LI, Sala MA, Brentegani LG, Carvalho TLL. Histometric study of socket healing after tooth extraction in rats treated with diclofenac. Brazilian Dental Journal. 2002;13(2):92-6. 
19. Habibovic P, Yuan H, van der Valk CM, Meijer G, van Blitterswijk CA, de Groot K. 3D microenvironment as essential element for osteoinduction by biomaterials. Biomaterials. 2005;26(17):3565-75.

20. Breuil V, Panaia-Ferrari P, Fontas E, Roux C, Kolta S, Eastell R, et al. Oxytocin, a new determinant of bone mineral density in post-menopausal women: analysis of the OPUS cohort. The Journal of Clinical Endocrinology \& Metabolism. 2014;99(4):E634-E41.

21. MacIntyre I, Zaidi M, Alam A, Datta HK, Moonga BS, Lidbury PS, et al. Osteoclastic inhibition: an action of nitric oxide not mediated by cyclic GMP. Proceedings of the National Academy of Sciences. 1991;88(7):293640 .

22. Cattaneo M, Chini B, Vicentini L. Oxytocin stimulates migration and invasion in human endothelial cells. British Journal of Pharmacology. 2008;153(4):728-36. 